\title{
A switching rule for exponential stability of switched recurrent neural networks with interval time-varying delay
}

\author{
Manlika Rajchakit', Piyapong Niamsup 2,3,4 and Grienggrai Rajchakit ${ }^{*}$
}

"Correspondence:

griengkrai@yahoo.com

1 Division of Mathematics and

Statistics, Faculty of Science, Maejo

University, Chiangmai, 50290,

Thailand

Full list of author information is

available at the end of the article

\begin{abstract}
This paper studies the problem for exponential stability of switched recurrent neural networks with interval time-varying delay. The time delay is a continuous function belonging to a given interval, but not necessarily differentiable. By constructing a set of argumented Lyapunov-Krasovskii functionals combined with the Newton-Leibniz formula, a switching rule for exponential stability of switched recurrent neural networks with interval time-varying delay is designed via linear matrix inequalities, and new sufficient conditions for the exponential stability of switched recurrent neural networks with interval time-varying delay via linear matrix inequalities (LMIs) are derived. A numerical example is given to illustrate the effectiveness of the obtained result.
\end{abstract}

Keywords: neural networks; switching design; exponential stability; interval time-varying delays; Lyapunov function; linear matrix inequalities

\section{Introduction}

In recent years, neural networks (especially recurrent neural networks, Hopfield neural networks, and cellular neural networks) have been successfully applied in many areas such as signal processing, image processing, pattern recognition, fault diagnosis, associative memory, and combinatorial optimization; see, for example, [1-6]. One of the best important works in these applications is to study the stability of the equilibrium point of neural networks. A major purpose is to find stability conditions i.e., the conditions for the stability of the equilibrium point of neural networks. The stability and control of recurrent neural networks with time delay have attracted considerable attention in recent years [1-10]. In many practical systems, it is desirable to design neural networks which are not only asymptotically or exponentially stable but can also guarantee an adequate level of system performance. In the area of control, signal processing, pattern recognition, and image processing, delayed neural networks have many useful applications. Some of these applications require the equilibrium points of the designed network to be stable. In both biological and artificial neural systems, time delays due to integration and communication are ubiquitous and often become a source of instability. The time delays in electronic neural networks are usually time-varying and sometimes vary violently with respect to time due to the finite switching speed of amplifiers and faults in the electrical circuitry. The Lyapunov-Krasovskii functional technique has been among the popular and effective

C) 2013 Rajchakit et al: licensee Springer. This is an Open Access article distributed under the terms of the Creative Commons Attribution License (http://creativecommons.org/licenses/by/2.0), which permits unrestricted use, distribution, and reproduction in any medium, provided the original work is properly cited. 
tools in the design of guaranteed cost controls for neural networks with time delay. Nevertheless, despite such diversity of results available, most existing works either assumed that the time delays are constant or differentiable [9-14]. To the best of our knowledge, a switching rule and exponential stability for switched recurrent neural networks with interval time-varying delay, non-differentiable time-varying delays have not been fully studied yet (see, e.g., $[4-9,13-25]$ and the references therein), and they are important in both theories and applications. This motivates our research.

In this paper, we investigate the exponential stability for a switched recurrent neural networks problem. The novel features here are that the delayed neural network under consideration is with various globally Lipschitz continuous activation functions, and the time-varying delay function is interval, non-differentiable. Based on constructing a set of augmented Lyapunov-Krasovskii functionals combined with Newton-Leibniz formula, a switching rule for exponential stability of switched recurrent neural networks with interval time-varying delay and new delay-dependent exponential stability criteria for switched recurrent neural networks with interval time-varying delay are established in terms of LMIs, which allow simultaneous computation of two bounds that characterize the exponential stability rate of the solution and can be easily determined by utilizing MATLABs LMI control toolbox.

The outline of the paper is as follows. Section 2 presents definitions and some wellknown technical propositions needed for the proof of the main result. LMI delaydependent exponential stability criteria for switched recurrent neural networks with interval time-varying delay criteria, a switching rule for exponential stability of switched recurrent neural networks with interval time-varying delay, and a numerical example showing the effectiveness of the result are presented in Section 3. The paper ends with conclusions and cited references.

\section{Preliminaries}

The following notations will be used in this paper. $\mathbb{R}^{+}$denotes the set of all real nonnegative numbers; $\mathbb{R}^{n}$ denotes the $n$-dimensional space with the scalar product $\langle x, y\rangle$ or $x^{T} y$ of two vectors $x, y$ and the vector norm $\|\cdot\| ; M^{n \times r}$ denotes the space of all matrices of $(n \times r)$-dimensions. $A^{T}$ denotes the transpose of matrix $A$; $A$ is symmetric if $A=A^{T}$; $I$ denotes the identity matrix; $\lambda(A)$ denotes the set of all eigenvalues of $A$; $\lambda_{\max }(A)=\max \{\operatorname{Re} \lambda ; \lambda \in \lambda(A)\}, \lambda_{\min }(A)=\min \{\operatorname{Re} \lambda ; \lambda \in \lambda(A)\} . x_{t}:=\left\{x(t+s): s \in\left[-h_{1}, 0\right]\right\}$, $\left\|x_{t}\right\|=\sup _{s \in\left[-h_{1}, 0\right]}\|x(t+s)\| ; C^{1}\left([0, t], \mathbb{R}^{n}\right)$ denotes the set of all $\mathbb{R}^{n}$-valued continuously differentiable functions on $[0, t] ; L_{2}\left([0, t], \mathbb{R}^{m}\right)$ denotes the set of all the $\mathbb{R}^{m}$-valued square integrable functions on $[0, t]$.

Matrix $A$ is called semi-positive definite $(A \geq 0)$ if $\langle A x, x\rangle \geq 0$ for all $x \in \mathbb{R}^{n} ; A$ is positive definite $(A>0)$ if $\langle A x, x\rangle>0$ for all $x \neq 0 ; A>B$ means $A-B>0$. The notation $\operatorname{diag}\{\cdots\}$ stands for a block-diagonal matrix. The symmetric term in a matrix is denoted by $*$.

Consider the following switched recurrent neural networks with interval time-varying delay:

$$
\begin{aligned}
& \dot{x}(t)=-A_{\gamma(x(t)} x(t)+W_{0 \gamma(x(t))} f(x(t))+W_{1 \gamma(x(t))} g(x(t-h(t))), \quad t \geq 0, \\
& x(t)=\phi(t), \quad t \in\left[-h_{1}, 0\right],
\end{aligned}
$$


where $x(t)=\left[x_{1}(t), x_{2}(t), \ldots, x_{n}(t)\right]^{T} \in \mathbb{R}^{n}$ is the state of the neural, $n$ is the number of neurals, and

$$
\begin{aligned}
& f(x(t))=\left[f_{1}\left(x_{1}(t)\right), f_{2}\left(x_{2}(t)\right), \ldots, f_{n}\left(x_{n}(t)\right)\right]^{T}, \\
& g(x(t))=\left[g_{1}\left(x_{1}(t)\right), g_{2}\left(x_{2}(t)\right), \ldots, g_{n}\left(x_{n}(t)\right)\right]^{T}
\end{aligned}
$$

are the activation functions; $\gamma(\cdot): R^{n} \rightarrow \mathcal{N}:=\{1,2, \ldots, N\}$ is the switching rule, which is a function depending on the state at each time and will be designed. A switching function is a rule which determines a switching sequence for a given switching system. Moreover, $\gamma(x(t))=j$ implies that the system realization is chosen as the $j$ th system, $j=1,2, \ldots, N$. It is seen that the system (2.1) can be viewed as an autonomous switched system in which the effective subsystem changes when the state $x(t)$ hits predefined boundaries.

$A_{j}=\operatorname{diag}\left(\bar{a}_{1 j}, \bar{a}_{2 j}, \ldots, \bar{a}_{n j}\right), \bar{a}_{i j}>0$ represents the self-feedback term; $W_{0 j}, W_{1 j}$ denote the connection weights, the discretely delayed connection weights, and the distributively delayed connection weight, respectively. The time-varying delay function $h(t)$ satisfies the condition

$$
0 \leq h_{0} \leq h(t) \leq h_{1}
$$

The initial functions $\phi(t) \in C^{1}\left(\left[-h_{1}, 0\right], R^{n}\right)$ with the norm

$$
\|\phi\|=\sup _{t \in\left[-h_{1}, 0\right]} \sqrt{\|\phi(t)\|^{2}+\|\dot{\phi}(t)\|^{2}} .
$$

In this paper, we consider various activation functions and assume that the activation functions $f(\cdot), g(\cdot)$ are Lipschitzian with the Lipschitz constants $f_{i}, e_{i}>0$ :

$$
\begin{aligned}
& \left|f_{i}\left(\xi_{1}\right)-f_{i}\left(\xi_{2}\right)\right| \leq f_{i}\left|\xi_{1}-\xi_{2}\right|, \quad i=1,2, \ldots, n, \forall \xi_{1}, \xi_{2} \in \mathbb{R} \\
& \left|g_{i}\left(\xi_{1}\right)-g_{i}\left(\xi_{2}\right)\right| \leq e_{i}\left|\xi_{1}-\xi_{2}\right|, \quad i=1,2, \ldots, n, \forall \xi_{1}, \xi_{2} \in \mathbb{R} .
\end{aligned}
$$

Definition 2.1 The zero solution of switched recurrent neural networks with interval time-varying delay (2.1) is $\alpha$-exponentially stable if there exist two positive numbers $\alpha>0$, $N>0$ such that every solution $x(t, \phi)$ satisfies the following condition:

$$
\|x(t, \phi)\| \leq N e^{-\alpha t}\|\phi\|, \quad \forall t \geq 0 .
$$

We introduce the following technical well-known propositions, which will be used in the proof of our results.

Proposition 2.1 (Schur complement lemma [26]) Given constant matrices $X, Y, Z$ with appropriate dimensions satisfying $X=X^{T}, Y=Y^{T}>0$. Then $X+Z^{T} Y^{-1} Z<0$ if and only if

$$
\left(\begin{array}{cc}
X & Z^{T} \\
Z & -Y
\end{array}\right)<0 .
$$


Proposition 2.2 (Integral matrix inequality [27]) For any symmetric positive definite matrix $M>0$, scalar $\sigma>0$ and vector function $\omega:[0, \sigma] \rightarrow \mathbb{R}^{n}$ such that the integrations concerned are well defined, the following inequality holds:

$$
\left(\int_{0}^{\sigma} \omega(s) d s\right)^{T} M\left(\int_{0}^{\sigma} \omega(s) d s\right) \leq \sigma\left(\int_{0}^{\sigma} \omega^{T}(s) M \omega(s) d s\right)
$$

\section{Main results}

Let us set

$$
\begin{aligned}
w_{11}= & -[P+\alpha I] A_{j}-A_{j}^{T}[P+\alpha I]+\sum_{i=0}^{1} G_{i}-P A_{j}-A_{j}^{T} P \\
& -\sum_{i=0}^{1} e^{-2 \alpha h_{i}} H_{i}+4 P F D_{0}^{-1} F P, \\
w_{12}= & P+A_{j} P, \quad w_{13}=e^{-2 \alpha h_{0}} H_{0}+A_{j} P, \\
w_{14}= & 2 e^{-2 \alpha h_{1}} H_{1}+A_{j} P, \quad w_{15}=P+A_{j} P, \\
w_{22}= & \sum_{i=0}^{1} W_{i j} D_{i} W_{i j}^{T}+\sum_{i=0}^{1} h_{i}^{2} H_{i}+\left(h_{1}-h_{0}\right) U-2 P, \\
w_{23}= & P, \quad w_{24}=P, \quad w_{25}=P, \\
w_{33}= & -e^{-2 \alpha h_{0}} G_{0}-e^{-2 \alpha h_{0}} H_{0}-e^{-2 \alpha h_{1}} U+\sum_{i=0}^{1} W_{i j} D_{i} W_{i j}^{T}, \\
w_{34}= & 0, \quad w_{35}=-2 \alpha h_{1} U, \\
& +h_{1}^{2} \lambda_{\max }\left[P^{-1}\left(\sum_{i=0}^{1} H_{i}\right) P^{-1}\right]+\left(h_{1}-h_{0}\right) \lambda_{\max }\left(P^{-1} U P^{-1}\right) . \\
w_{44}= & \sum_{i=0}^{1} W_{i j} D_{i} W_{i j}^{T}-e^{-2 \alpha h_{1}} U-e^{-2 \alpha h_{1}} G_{1}-e^{-2 \alpha h_{1}} H_{1}, \\
w_{45}= & e^{-2 \alpha h_{1}} U, \\
w_{55}= & \sum_{i=0}^{1} W_{i j} D_{i} W_{i j}^{T}-e^{-2 \alpha h_{1}} U-e^{-2 \alpha h_{2}} U+4 P E D_{1}^{-1} E P, \\
E= & \operatorname{diag}\left\{e_{i}, i=1, \ldots, n\right\}, \quad F=\operatorname{diag}\left\{f_{i}, i=1, \ldots, n\right\}, \\
\lambda_{1}= & \lambda_{\min }\left(P^{-1}\right), \\
\lambda_{2}= & \lambda_{\max }\left(P^{-1}\right)+h_{0} \lambda_{\max }\left[P^{-1}\left(\sum_{i=0}^{1} G_{i}\right) P^{-1}\right]
\end{aligned}
$$

Theorem 3.1 The zero solution of the switched recurrent neural networks with interval time-varying delay (2.1) is $\alpha$-exponentially stable if there exist a positive number $\alpha>0$, symmetric positive definite matrices $P, U, G_{0}, G_{1}, H_{0}, H_{1}$, and diagonal positive definite 
matrices $D_{i}, i=0,1$ satisfying the following LMIs:

$$
\left[\begin{array}{ccccc}
w_{11} & w_{12} & w_{13} & w_{14} & w_{15} \\
* & w_{22} & w_{23} & w_{24} & w_{25} \\
* & * & w_{33} & w_{34} & w_{35} \\
* & * & * & w_{44} & w_{45} \\
* & * & * & * & w_{55}
\end{array}\right]<0, \quad j=1,2, \ldots, N
$$

the switching rule is chosen as $\gamma(x(t))=j$. Moreover, the solution $x(t, \phi)$ of the system satisfies

$$
\|x(t, \phi)\| \leq \sqrt{\frac{\lambda_{2}}{\lambda_{1}}} e^{-\alpha t}\|\phi\|, \quad \forall t \geq 0 .
$$

Proof Let $Y=P^{-1}, y(t)=Y x(t)$. We consider the following Lyapunov-Krasovskii functional:

$$
\begin{aligned}
& V\left(t, x_{t}\right)=\sum_{i=1}^{6} V_{i}\left(t, x_{t}\right), \\
& V_{1}=x^{T}(t) Y x(t), \\
& V_{2}=\int_{t-h_{0}}^{t} e^{2 \alpha(s-t)} x^{T}(s) Y G_{0} Y x(s) d s, \\
& V_{3}=\int_{t-h_{1}}^{t} e^{2 \alpha(s-t)} x^{T}(s) Y G_{1} Y x(s) d s, \\
& V_{4}=h_{0} \int_{-h_{0}}^{0} \int_{t+s}^{t} e^{2 \alpha(\tau-t)} \dot{x}^{T}(\tau) Y H_{0} Y \dot{x}(\tau) d \tau d s, \\
& V_{5}=h_{1} \int_{-h_{1}}^{0} \int_{t+s}^{t} e^{2 \alpha(\tau-t)} \dot{x}^{T}(\tau) Y H_{1} Y \dot{x}(\tau) d \tau d s, \\
& V_{6}=\left(h_{1}-h_{0}\right) \int_{t-h_{1}}^{t-h_{0}} \int_{t+s}^{t} e^{2 \alpha(\tau-t)} \dot{x}^{T}(\tau) Y U Y \dot{x}(\tau) d \tau d s .
\end{aligned}
$$

It is easy to check that

$$
\lambda_{1}\|x(t)\|^{2} \leq V\left(t, x_{t}\right) \leq \lambda_{2}\left\|x_{t}\right\|^{2}, \quad \forall t \geq 0 .
$$

Taking the derivative of $V_{1}$, we have

$$
\begin{aligned}
\dot{V}_{1} & =2 x^{T}(t) Y \dot{x}(t) \\
& =y^{T}(t)\left[-P A_{j}^{T}-A_{j} P\right] y(t)+2 y^{T}(t) W_{0 j} f(\cdot) P y(t)+2 y^{T}(t) W_{1 j} g(\cdot) P y(t) ; \\
\dot{V}_{2} & =y^{T}(t) G_{0} y(t)-e^{-2 \alpha h_{0}} y^{T}\left(t-h_{0}\right) G_{0} y\left(t-h_{0}\right)-2 \alpha V_{2} ; \\
\dot{V}_{3} & =y^{T}(t) G_{1} y(t)-e^{-2 \alpha h_{1}} y^{T}\left(t-h_{1}\right) G_{1} y\left(t-h_{1}\right)-2 \alpha V_{3} ; \\
\dot{V}_{4} & =h_{0}^{2} \dot{y}^{T}(t) H_{0} \dot{y}(t)-h_{1} e^{-2 \alpha h_{0}} \int_{t-h_{0}}^{t} \dot{x}^{T}(s) H_{0} \dot{x}(s) d s-2 \alpha V_{4} ;
\end{aligned}
$$




$$
\begin{aligned}
& \dot{V}_{5}=h_{1}^{2} \dot{y}^{T}(t) H_{1} \dot{y}(t)-h_{1} e^{-2 \alpha h_{1}} \int_{t-h_{1}}^{t} \dot{y}^{T}(s) H_{1} \dot{y}(s) d s-2 \alpha V_{5} ; \\
& \dot{V}_{6}=\left(h_{1}-h_{0}\right)^{2} \dot{y}^{T}(t) U \dot{y}(t)-\left(h_{1}-h_{0}\right) e^{-2 \alpha h_{1}} \int_{t-h_{1}}^{t-h_{0}} \dot{y}^{T}(s) U \dot{y}(s) d s-2 \alpha V_{6} .
\end{aligned}
$$

Applying Proposition 2.2 and the Leibniz-Newton formula

$$
\int_{s}^{t} \dot{y}(\tau) d \tau=y(t)-y(s)
$$

we have, for $i=0,1$,

$$
\begin{aligned}
-h_{i} \int_{t-h_{i}}^{t} \dot{y}^{T}(s) H_{i} \dot{y}(s) d s \leq & -\left[\int_{t-h_{i}}^{t} \dot{y}(s) d s\right]^{T} H_{i}\left[\int_{t-h_{i}}^{t} \dot{y}(s) d s\right] \\
\leq & -[y(t)-y(t-h(t))]^{T} H_{i}[y(t)-y(t-h(t))] \\
= & -y^{T}(t) H_{i} y(t)+2 y^{T}(t) H_{i} y(t-h(t)) \\
& -y^{T}\left(t-h_{i}\right) H_{i} y\left(t-h_{i}\right) .
\end{aligned}
$$

Note that

$$
\int_{t-h_{1}}^{t-h_{0}} \dot{y}^{T}(s) U \dot{y}(s) d s=\int_{t-h_{1}}^{t-h(t)} \dot{y}^{T}(s) U \dot{y}(s) d s+\int_{t-h(t)}^{t-h_{0}} \dot{y}^{T}(s) U \dot{y}(s) d s .
$$

Applying Proposition 2.2 gives

$$
\begin{aligned}
{\left[h_{1}-h(t)\right] \int_{t-h_{1}}^{t-h(t)} \dot{y}^{T}(s) U \dot{y}(s) d s } & \geq\left[\int_{t-h_{1}}^{t-h(t)} \dot{y}(s) d s\right]^{T} U\left[\int_{t-h_{1}}^{t-h(t)} \dot{y}(s) d s\right] \\
& \geq\left[y(t-h(t))-y\left(t-h_{1}\right)\right]^{T} U\left[y(t-h(t))-y\left(t-h_{1}\right)\right] .
\end{aligned}
$$

Since $h_{1}-h(t) \leq h_{1}-h_{0}$, we have

$$
\left[h_{1}-h_{0}\right] \int_{t-h_{1}}^{t-h(t)} \dot{y}^{T}(s) U \dot{y}(s) d s \geq\left[y(t-h(t))-y\left(t-h_{1}\right)\right]^{T} U\left[y(t-h(t))-y\left(t-h_{1}\right)\right],
$$

then

$$
-\left[h_{1}-h_{0}\right] \int_{t-h_{1}}^{t-h(t)} \dot{y}^{T}(s) U \dot{y}(s) d s \leq-\left[y(t-h(t))-y\left(t-h_{1}\right)\right]^{T} U\left[y(t-h(t))-y\left(t-h_{1}\right)\right] .
$$

Similarly, we have

$$
-\left(h_{1}-h_{0}\right) \int_{t-h(t)}^{t-h_{0}} \dot{y}^{T}(s) U \dot{y}(s) d s \leq-\left[y\left(t-h_{0}\right)-y(t-h(t))\right]^{T} U\left[y\left(t-h_{0}\right)-y(t-h(t))\right] .
$$

Then we have

$$
\begin{aligned}
\dot{V}(\cdot)+2 \alpha V(\cdot) \leq & y^{T}(t)\left[-P A_{j}^{T}-A_{j} P\right] y(t)+2 y^{T}(t) W_{0 j} f(\cdot) P y(t) \\
& +2 y^{T}(t) W_{1 j} g(\cdot) P y(t)+y^{T}(t)\left(\sum_{i=0}^{1} G_{i}\right) y(t)+2 \alpha\langle P y(t), y(t)\rangle
\end{aligned}
$$




$$
\begin{aligned}
& +\dot{y}^{T}(t)\left(\sum_{i=0}^{1} h_{i}^{2} H_{i}\right) \dot{y}(t)+\left(h_{1}-h_{0}\right) \dot{y}^{T}(t) U \dot{y}(t) \\
& -\sum_{i=0}^{1} e^{-2 \alpha h_{i}} y^{T}\left(t-h_{i}\right) G_{i} y\left(t-h_{i}\right) \\
& -e^{-2 \alpha h_{0}}\left[y(t)-y\left(t-h_{0}\right)\right]^{T} H_{0}\left[y(t)-y\left(t-h_{0}\right)\right] \\
& -e^{-2 \alpha h_{1}}\left[y(t)-y\left(t-h_{1}\right)\right]^{T} H_{1}\left[y(t)-y\left(t-h_{1}\right)\right] \\
& -e^{-2 \alpha h_{1}}\left[y(t-h(t))-y\left(t-h_{1}\right)\right]^{T} U\left[y(t-h(t))-y\left(t-h_{1}\right)\right] \\
& -e^{-2 \alpha h_{1}}\left[y\left(t-h_{0}\right)-y(t-h(t))\right]^{T} U\left[y\left(t-h_{0}\right)-y(t-h(t))\right] .
\end{aligned}
$$

Using equation (2.1),

$$
P \dot{y}(t)+A_{j} P y(t)-W_{0 j} f(\cdot)-W_{1 j} g(\cdot)=0,
$$

and multiplying both sides by $\left[2 y(t),-2 \dot{y}(t), 2 y\left(t-h_{0}\right), 2 y\left(t-h_{1}\right), 2 y(t-h(t))\right]^{T}$, we have

$$
\begin{aligned}
& 2 y^{T}(t) P \dot{y}(t)+2 y^{T}(t) A_{j} P y(t)-2 y^{T}(t) W_{0 j} f(\cdot)-2 y^{T}(t) W_{1 j} g(\cdot)=0, \\
& -2 \dot{y}^{T}(t) P \dot{y}(t)-2 \dot{y}^{T}(t) A_{j} P y(t)+2 \dot{y}^{T}(t) W_{0 j} f(\cdot)+2 \dot{y}^{T}(t) W_{1 j} g(\cdot)=0, \\
& 2 y^{T}\left(t-h_{0}\right) P \dot{y}(t)+2 y^{T}\left(t-h_{0}\right) A_{j} P y(t) \\
& \quad-2 y^{T}\left(t-h_{0}\right) W_{0 j} f(\cdot)-2 y^{T}\left(t-h_{0}\right) W_{1 j} g(\cdot)=0, \\
& 2 y^{T}\left(t-h_{1}\right) P \dot{y}(t)+2 y^{T}\left(t-h_{1}\right) A_{j} P y(t)-2 y^{T}\left(t-h_{1}\right) W_{0 j} f(\cdot)-2 y^{T}\left(t-h_{1}\right) W_{1 j} g(\cdot)=0, \\
& 2 y^{T}(t-h(t)) P \dot{y}(t)+2 y^{T}(t-h(t)) A_{j} P y(t) \\
& \quad-2 y^{T}(t-h(t)) W_{0 j} f(\cdot)-2 y^{T}(t-h(t)) W_{1 j} g(\cdot)=0 .
\end{aligned}
$$

Adding all the zero items of (3.5) into (3.4) for the following estimations:

$$
\begin{aligned}
& 2\left\langle W_{0 j} f(x), y\right\rangle \leq\left\langle W_{0 j} D_{0} W_{0 j}^{T} y, y\right\rangle+\left\langle D_{0}^{-1} f(x), f(x)\right\rangle, \\
& 2\left\langle W_{1 j} g(z), y\right\rangle \leq\left\langle W_{1 j} D_{1} W_{1 j}^{T} y, y\right\rangle+\left\langle D_{1}^{-1} g(z), g(z)\right\rangle, \\
& 2\left\langle D_{0}^{-1} f(x), f(x)\right\rangle \leq\left\langle F D_{0}^{-1} F x, x\right\rangle, \\
& 2\left\langle D_{1}^{-1} g(z), g(z)\right\rangle \leq\left\langle E D_{1}^{-1} E z, z\right\rangle,
\end{aligned}
$$

we obtain

$$
\dot{V}(\cdot)+2 \alpha V(\cdot) \leq \zeta^{T}(t) \mathcal{E}_{j} \zeta(t)
$$

where $\zeta(t)=\left[y^{T}(t), \dot{y}^{T}(t), y^{T}\left(t-h_{0}\right), y^{T}\left(t-h_{1}\right), y^{T}(t-h(t))\right]^{T}$, and

$$
\mathcal{E}_{j}=\left[\begin{array}{ccccc}
w_{11} & w_{12} & w_{13} & w_{14} & w_{15} \\
* & w_{22} & w_{23} & w_{24} & w_{25} \\
* & * & w_{33} & w_{34} & w_{35} \\
* & * & * & w_{44} & w_{45} \\
* & * & * & * & w_{55}
\end{array}\right], \quad j=1,2, \ldots, N .
$$


Therefore, by condition (3.1), we obtain from (3.6) that

$$
\dot{V}\left(t, x_{t}\right) \leq-2 \alpha V\left(t, x_{t}\right), \quad \forall t \geq 0
$$

Integrating both sides of (3.7) from 0 to $t$, we obtain

$$
V\left(t, x_{t}\right) \leq V(\phi) e^{-2 \alpha t}, \quad \forall t \geq 0
$$

Furthermore, taking condition (3.2) into account, we have

$$
\lambda_{1}\|x(t, \phi)\|^{2} \leq V\left(x_{t}\right) \leq V(\phi) e^{-2 \alpha t} \leq \lambda_{2} e^{-2 \alpha t}\|\phi\|^{2},
$$

then

$$
\|x(t, \phi)\| \leq \sqrt{\frac{\lambda_{2}}{\lambda_{1}}} e^{-\alpha t}\|\phi\|, \quad t \geq 0
$$

which concludes the exponential stability of (2.1). This completes the proof of the theorem.

Example 3.1 Consider the switched recurrent neural networks with interval time-varying delay (2.1) for $j=2$, where

$$
\begin{aligned}
& A_{1}=\left[\begin{array}{cc}
0.1 & 0 \\
0 & 0.3
\end{array}\right], \quad A_{2}=\left[\begin{array}{cc}
0.2 & 0 \\
0 & 0.4
\end{array}\right], \\
& W_{01}=\left[\begin{array}{cc}
-0.1 & 0.3 \\
0.2 & -0.8
\end{array}\right], \quad W_{02}=\left[\begin{array}{cc}
-0.7 & 0.3 \\
0.4 & -0.9
\end{array}\right], \\
& W_{11}=\left[\begin{array}{cc}
-0.4 & 0.2 \\
0.3 & -0.3
\end{array}\right], \quad W_{12}=\left[\begin{array}{cc}
-0.2 & 0.3 \\
0.1 & -0.4
\end{array}\right], \\
& E=\left[\begin{array}{cc}
0.2 & 0 \\
0 & 0.4
\end{array}\right], \quad F=\left[\begin{array}{cc}
0.3 & 0 \\
0 & 0.5
\end{array}\right], \\
& \left\{\begin{array}{l}
h(t)=0.1+1.2 \sin ^{2} t \quad \text { if } t \in \mathcal{I}=\bigcup_{k \geq 0}[2 k \pi,(2 k+1) \pi], \\
h(t)=0
\end{array}\right.
\end{aligned}
$$

Note that $h(t)$ is non-differentiable, therefore, the stability criteria proposed in $[5-9,11-$ $14,17-25]$ are not applicable to this system. We choose that $\alpha=0.4, h_{0}=0.1, h_{1}=1.3$. By using the Matlab LMI toolbox, we can solve linear matrix inequalities for $P, U, G_{0}, G_{1}$, $D_{0}, D_{1}, H_{0}$ and $H_{1}$ which satisfy the conditions (3.1) in Theorem 3.1. A set of solutions is as follows:

$$
\begin{array}{ll}
P=\left[\begin{array}{cc}
1.5219 & -0.3659 \\
-0.3659 & 2.2398
\end{array}\right], \quad U=\left[\begin{array}{cc}
3.1239 & -0.2365 \\
-0.2365 & 3.0123
\end{array}\right], \\
G_{0}=\left[\begin{array}{ll}
1.3225 & 0.0258 \\
0.0258 & 1.2698
\end{array}\right], & G_{1}=\left[\begin{array}{cc}
2.2368 & 0.0148 \\
0.0148 & 3.1121
\end{array}\right],
\end{array}
$$




$$
\begin{array}{ll}
H_{0}=\left[\begin{array}{ll}
2.2189 & 0.1238 \\
0.1238 & 1.2368
\end{array}\right], & H_{1}=\left[\begin{array}{ll}
2.3225 & 0.0369 \\
0.0369 & 2.1897
\end{array}\right], \\
D_{1}=\left[\begin{array}{ll}
1.2398 & 0.3659 \\
0.3659 & 1.8935
\end{array}\right], & D_{2}=\left[\begin{array}{ll}
2.3641 & 0.0593 \\
0.0593 & 1.2380
\end{array}\right] .
\end{array}
$$

By Theorem 3.1, the switched recurrent neural networks with interval time-varying delay are exponentially stable and the switching rule is chosen as $\gamma(x(t))=j$. Moreover, the solution $x(t, \phi)$ of the system satisfies

$$
\|x(t, \phi)\| \leq 12.6984 e^{-0.4 t}\|\phi\|, \quad \forall t \geq 0 .
$$

\section{Conclusion}

In this paper, the problem of exponential stability for switched recurrent neural networks with interval non-differentiable time-varying delay has been studied. By constructing a set of time-varying Lyapunov-Krasovskii functional combined with Newton-Leibniz formula, a switching rule for exponential stability of switched recurrent neural networks with interval time-varying delay has been presented and new sufficient conditions for the exponential stability for the system have been derived in terms of LMIs.

\section{Competing interests}

The authors declare that they have no competing interests.

\section{Authors' contributions}

The authors contributed equally and significantly in writing this paper. The authors read and approved the final manuscript.

\section{Author details}

${ }^{1}$ Division of Mathematics and Statistics, Faculty of Science, Maejo University, Chiangmai, 50290, Thailand. ${ }^{2}$ Deparment of Mathematics, Faculty of Science, Chiang Mai University, Chiangmai, 52000, Thailand. ${ }^{3}$ Center of Excellence in Mathematics, CHE, Si Ayutthaya Rd., Bangkok, 10400, Thailand. ${ }^{4}$ Düsternbrooker Weg 20, Kiel, 24105, Germany.

\section{Acknowledgements}

This work was supported by the Office of Agricultural Research and Extension Maejo University Chiang Mai Thailand, the National Research Council of Thailand, the Thailand Research Fund Grant, the Higher Education Commission and Faculty of Science, Maejo University, Thailand. The authors thank anonymous reviewers for valuable comments and suggestions, which allowed us to improve the paper.

Received: 22 September 2012 Accepted: 17 January 2013 Published: 28 February 2013

\section{References}

1. Hopfield, JJ: Neural networks and physical systems with emergent collective computational abilities. Proc. Natl. Acad. Sci. USA 79, 2554-2558 (1982)

2. Ratchagit, K: Asymptotic stability of delay-difference system of Hopfield neural networks via matrix inequalities and application. Int. J. Neural Syst. 17, 425-430 (2007)

3. Kevin, G: An Introduction to Neural Networks. CRC Press, Boca Raton (1997)

4. $\mathrm{Wu}, \mathrm{M}, \mathrm{He}$, Y, She, JH: Stability Analysis and Robust Control of Time-Delay Systems. Springer, Berlin (2010)

5. Arik, S: An improved global stability result for delayed cellular neural networks. IEEE Trans. Circuits Syst. 499, 1211-1218 (2002)

6. He, Y, Wang, QG, Wu, M: LMI-based stability criteria for neural networks with multiple time-varying delays. Physica D $112,126-131(2005)$

7. Kwon, OM, Park, JH: Exponential stability analysis for uncertain neural networks with interval time-varying delays. Appl. Math. Comput. 212, 530-541 (2009)

8. Phat, VN, Trinh, H: Exponential stabilization of neural networks with various activation functions and mixed time-varying delays. IEEE Trans. Neural Netw. 21, 1180-1185 (2010)

9. Botmart, T, Niamsup, P: Robust exponential stability and stabilizability of linear parameter dependent systems with delays. Appl. Math. Comput. 217, 2551-2566 (2010)

10. Fridman, E, Orlov, Y: Exponential stability of linear distributed parameter systems with time-varying delays. Automatica 45, 194-201 (2009)

11. Xu, S, Lam, J: A survey of linear matrix inequality techniques in stability analysis of delay systems. Int. J. Syst. Sci. 39(12), 1095-1113(2008) 
12. Xie, JS, Fan, BQ, Young, SL, Yang, J: Guaranteed cost controller design of networked control systems with state delay. Acta Autom. Sin. 33, 170-174 (2007)

13. Yu, L, Gao, F: Optimal guaranteed cost control of discrete-time uncertain systems with both state and input delays. J. Franklin Inst. 338, 101-110 (2001)

14. Park, JH, Kwon, OM, Lee, SM, Won, SC: On robust $H_{\infty}$ filter design for uncertain neural systems: LMI optimization approach. Appl. Math. Comput. 159, 625-639 (2004)

15. Park, JH: Further result on asymptotic stability criterion of cellular neural networks with time-varying discrete and distributed delays. Appl. Math. Comput. 182, 1661-1666 (2006)

16. Ratchagit, K, Phat, VN: Robust stability and stabilization of linear polytopic delay-difference equations with interval time-varying delays. Neural Parallel Sci. Comput. 19, 361-372 (2011)

17. Phat, VN, Ratchagit, K: Stability and stabilization of switched linear discrete-time systems with interval time-varying delay. Nonlinear Anal. Hybrid Syst. 5, 605-612 (2011)

18. Tian, L, Liang, J, Cao, J: Robust observer for discrete-time Markovian jumping neural networks with mixed mode-dependent delays. Nonlinear Dyn. 67, 47-61 (2012)

19. Rajchakit, M, Rajchakit, G: Mean square exponential stability of stochastic switched system with interval time-varying delays. Abstr. Appl. Anal. 2012, Article ID 623014 (2012). doi:10.1155/2012/623014

20. Phat, VN, Kongtham, Y, Ratchagit, K: LMI approach to exponential stability of linear systems with interval time-varying delays. Linear Algebra Appl. 436, 243-251 (2012)

21. $\mathrm{Wu}, \mathrm{H}, \mathrm{Li}, \mathrm{N}, \mathrm{Wang}, \mathrm{K}, \mathrm{Xu}, \mathrm{G}, \mathrm{Guo}, \mathrm{Q}$ : Global robust stability of switched interval neural networks with discrete and distributed time-varying delays of neural type. Math. Probl. Eng. 2012, Article ID 361871 (2012) doi:10.1155/2012/361871

22. $\mathrm{Xu}, \mathrm{H}, \mathrm{Wu}, \mathrm{H}, \mathrm{Li}, \mathrm{N}$ : Switched exponential state estimation and robust stability for interval neural networks with discrete and distributed time delays. Abstr. Appl. Anal. 2012, Article ID 103542 (2012). doi:10.1155/2012/103542

23. Rajchakit, M, Niamsup, P, Rojsiraphisal, T, Rajchakit, G: Delay-dependent guaranteed cost controller design for uncertain neural networks with interval time-varying delay. Abstr. Appl. Anal. 2012, Article ID 587426 (2012). doi:10.1155/2012/587426

24. Zhang, H, Wang, Z, Liu, D: Robust exponential stability of recurrent neural networks with multiple time-varying delays. IEEE Trans. Circuits Syst. II, Express Briefs 54(8), 730-734 (2007)

25. Gau, R-S, Lien, C-H, Hsieh, J-G: Novel stability conditions for interval delayed neural networks with multiple time-varying delays. Int. J. Innov. Comput. Inf. Control 7(1), 433-444 (2011)

26. Boyd, S, El Ghaoui, L, Feron, E, Balakrishnan, V: Linear Matrix Inequalities in System and Control Theory. SIAM, Philadelphia (1994)

27. Gu, K, Kharitonov, V, Chen, J: Stability of Time-Delay Systems. Birkhäuser, Berlin (2003)

doi:10.1186/1687-1847-2013-44

Cite this article as: Rajchakit et al.: A switching rule for exponential stability of switched recurrent neural networks with interval time-varying delay. Advances in Difference Equations 2013 2013:44.

\section{Submit your manuscript to a SpringerOpen ${ }^{\ominus}$ journal and benefit from:}

- Convenient online submission

- Rigorous peer review

- Immediate publication on acceptance

Open access: articles freely available online

- High visibility within the field

- Retaining the copyright to your article 